\title{
The Extent to which Students use their Online Written Feedback while Taking Online Classes
}

\author{
Timothy Sawicki, Shawn O'Rourke \\ Canisius College, Buffalo, NY
}

\begin{abstract}
Quality written feedback is one of the factors that make up a good online class. The current research studied eight classes of students using two different levels of feedback prompts. The results showed that the Level 1 feedback prompt resulted in 39\% of students reading their feedback. The Level 2 feedback prompt showed a $44 \%$ overall feedback reading rate for students taking online classes. The 44\% of students In the Level 2 feedback reading their online written feedback is a $10 \%$ increase in students' reading their online written feedback from $34 \%$ found in an original study [9]. Further analysis were completed related to when the students most read their online written feedback in the course and whether there is any difference in reading rates for minor versus major assignments. The current research main focus is to analyze ways to improve student feedback reading rate. An anecdotal third group were studied to provide future directions for the research.
\end{abstract}

\section{Introduction}

Instructors spend a great deal of time writing online written feedback for their students to read. Careless [1] wrote about the importance of written feedback in student work and how under-researched it is. The study concluded that instructors thought their feedback was very important whereas students did not feel the feedback was as important as instructors thought it was. The thought that students do not find feedback important or useful could have implications for the current research. Ramsden [8] stated that quality teaching requires effective comments on students' work. Feedback in itself is a complex aspect to study regarding online teaching and since students question its usefulness, studies are needed to address this concern. There is a need in the research to validate the importance of instructor feedback in the online setting [4] and [5]. Zhu [12] stated that student learning is not automatic in online learning and that teacher intervention through feedback is an important part of online teaching.

In-depth, timely and quality written feedback is needed for good online teaching [2]. In a recent study, fifty-eight percent of students felt feedback would be better if it was timely and if there was more of it [10]. Mandernach and Garrett [6] stated that online feedback is more than just a written statement after a student assignment. It helps students determine where they should direct their efforts, whether they are on track with their work level or not and whether they are meeting the teachers' expectations. Feedback is of critical value to student learning. Student comments on feedback type and frequency have been made but how often do students actually read their online feedback is a question needed to be further addressed.

The quality and quantity of feedback needs to be relevant, specific and understandable and the timing of feedback is very crucial. For most students, the time-frame within which it is possible to learn from, and apply, feedback from one piece of work to another is narrow, particularly within a single module. Changes need to occur to move away from the frustrating cycle of reams of unread feedback [7]. This leads to the question if students often miss reading their online feedback, are there ways to improve the likelihood students will read their online feedback. The current research addresses this question.

Coll, Rochera and de Gispert [3] proposed an analytic framework for how and when feedback is best given for knowledge building in collaborative online settings. The authors stated more research is needed regarding feedback patterns in varied higher education programs in different countries.

Recent research by Sawicki and O'Rourke [9] showed that overall $34 \%$ of students read their online written feedback without any prompts from the instructor. The study also showed that students read their major assignments at a slightly higher rate than minor assignments and they read feedback earlier in the course at more frequent rate than later assignments. This lead to the current research which is studying ways to improve the student reading rate of their online written feedback.

\section{Method}

The current research looked at two ways to increase students reading their written online 
feedback. The study detailed feedback reading patterns using eight classes of graduate courses with a total number of 94 students. Six classes of students totaling 61 students were provided Level 1 feedback prompts. The instructor left a total of 638 online written feedbacks for all Level 1 students. Level 1 feedback prompt was defined as the instructor providing and directing students to read their online written feedback a few times throughout the course through the Announcements page. In the current study, the Announcements page specifically stated:

"Written online feedback is now available for Module-Assignment \#1... you can view that in your drop box. Viewing all written online feedback will help you complete the next module(s)-assignment(s)... if you ever have any questions about feedback or the course you can email me any time."

The research studied two graduate classes totaling 33 students who were provided Level 2 feedback prompts. A total of 363 online written feedbacks were left for Level 2 students. Level 2 feedback is defined as making students aware of their written online feedback as in Level 1 (see above Announcement post) plus adding a requirement that students must read their online feedback in their course and reflect on their feedback for a numerical grade. The students were given points for reading and reflecting on their feedback. For the Level 2 research group a small three-point assignment was used to direct students to read and reflect on their instructor feedback taken from a previous assignment. This was done early in the course, (module 2) so students would be using their online written feedback soon after they began the course. This hopefully would accustom the students to going to their drop box and reading their feedback instead for just reading their numerical grade in the online grade book.

It is hypothesized that the Level 2 feedback prompts, especially in Module 2 will raise the student feedback reading rate to $100 \%$ since their grades are affected by it. Students would lose three points on the semester (out of 100 points) if they did not read and reflect on their written online feedback left by the instructor.

A third group of students (which would be Level 3 feedback prompt) were studied anecdotally by the researchers. Twenty-four students from the MSA (Master of Sport Administration) in one course were withheld grades on their assignments which were usually posted to the grade book. By doing this the students were forced to go into their assignment drop box to read their online written feedback and read their numerical grade which was included with the qualitative written feedback. The numerical grade was posted with their written feedback and was not posted to the grade book. It was speculated this would achieve $100 \%$ feedback read rate since this was the only way students could get any feedback on the course assignments they handed in.

\section{Analysis of Findings}

An original study by Sawicki and O'Rourke [9] showed that $34 \%$ of student read their online written feedback across minor and major assignments when the students were not prompted to read their online written feedback. Such a low percentage may surprise instructors considering the time and effort instructors put in to write feedback for their students. Further, studies on good online teaching show that quality written feedback is important yet students do not always view feedback as crucial [1].

The current research set out to improve the rate of students read their online written feedback by providing some students with Level 1 prompts and some students with Level 2 prompts. The results showed that the Level 1 prompts improved online feedback reading rates to $39 \%$ overall from the original study by Sawicki and O'Rourke [9] which showed a $34 \%$ reading rate across all major and minor assignments. In the current study, Level 1 feedback prompts on minor assignments were read at a rate of 36\% (199 feedbacks read/ 559 feedbacks left by the instructor). Major assignments were read at a rate of 66\% (40 feedbacks read/ 61 feedbacks left by the instructor). This represented a $39 \%$ overall feedback reading rate $(239$ feedbacks read/ 620 feedbacks left by the instructor). This is a $5 \%$ increase from original study (Sawicki and O'Rourke [9], which used no direct prompts. The current Level 1 feedback prompt group showed a 66\% major assignment feedbackreading rate. This indicates that availing students, the knowledge that is feedback is available and prompting them that they should read it convinces them to read the feedback, especially on major assignments. The Level 1 prompt feedback groups showed a 66\% feedback-reading rate when the assignments are worth a great deal of their grades (i.e., 30-40\% of their final grade). The feedback reading rate is much lower (36\%) on minor assignments worth $3-5 \%$ of their grade across modules. The overall reading rate of $39 \%$ was an improvement over the $34 \%$ feedback reading rate found in the original study (Sawicki and O'Rourke [9] when no feedback prompts were provided.

The results showed that the Level 2 prompts improved online feedback reading rates to $44 \%$ overall from the original study by Sawicki and O'Rourke [9] which showed a $34 \%$ reading rate and improved on the $39 \%$ Level 1 prompt group in the current study. The Level 2 feedback prompt students were required to read the feedback on one of their early module assignments and hand in a feedback reflection for a grade. In the Level 2 feedback prompt group, minor assignments were read at a rate of $44 \%$ (145 feedbacks read/ 330 feedbacks left by the 
instructor). Major assignments were read at a rate of $39 \%$ (13 feedbacks read/ 33 feedbacks left by the instructor). This represented a $44 \%$ overall feedback reading rate $(158$ feedbacks read/ 363 feedbacks left by the instructor). The $39 \%$ for major assignments read is lower than expected and is most likely attributed to a small sample size. Work is underway to increase the Level 2 control group size and add to the current research. It was interesting to note that across the first five modules that the Level 2 group reviewed their feedback at a rate of 58\% (95 feedbacks read/ 165 feedbacks left by the instructor). Further results showed that Level 2 group assignment (module 2) required students to read the feedback for a numerical grade had an $82 \%$ feedback-reading rate. We had speculated and expected a $100 \%$ feedback- reading rate on module two when a student grade is part of it. However, it should be pointed out that it is only a minor assignment value (three points out of 100-point semester total). This indicated that requiring students to read feedback for grades might not always create $100 \%$ compliance. Table 1 demonstrates the tallies and percentage of student feedback (FB) read across all course modules and for final project/ the final exam for the Level 1 Feedback prompt group. Table 2 demonstrates the tallies and percentage of student feedback $(\mathrm{FB})$ read across all course modules and for final project/ the final exam for the Level 2 Feedback prompt group.

Table 1. Percentage of Feedback Read across Course Assignments- Level 1 Prompt

\begin{tabular}{|c|c|c|c|c|c|c|c|c|c|c|c|}
\hline \# of Students & Mod.1 & Mod.2 & Mod.3 & Mod.4 & Mod.5 & Mod.6 & Mod.7 & Mod.8 & Mod.9 & Mod.10 & Final Exam/Project \\
\hline $\begin{array}{l}\text { Total } / 6 \\
\text { Course } 1\end{array}$ & 3 & 2 & 2 & 2 & 2 & 2 & 1 & 1 & 1 & 0 & 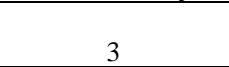 \\
\hline$\%$ FB Read & 50 & 33 & 33 & 33 & 33 & 33 & 17 & 17 & 17 & 17 & 50 \\
\hline $\begin{array}{l}\text { Total } / 13 \\
\text { Course } 2\end{array}$ & 7 & 7 & 7 & 8 & 5 & 4 & 5 & 6 & 6 & 3 & 10 \\
\hline$\%$ FB Read & 54 & 54 & 54 & 62 & 39 & 31 & 39 & 46 & 46 & 23 & 77 \\
\hline $\begin{array}{l}\text { Total/9 } \\
\text { Course } 3 \\
\end{array}$ & 7 & 5 & 4 & 4 & 4 & 5 & 2 & 2 & 3 & 2 & 5 \\
\hline$\%$ FB Read & 78 & 56 & 44 & 44 & 44 & 56 & 22 & 22 & 33 & 22 & 56 \\
\hline $\begin{array}{l}\text { Total } / 6 \\
\text { Course } 4\end{array}$ & 2 & 4 & 3 & 2 & 1 & 2 & 3 & & & & 3 \\
\hline$\%$ FB Read & 33 & 67 & 50 & 33 & 17 & 33 & 50 & & & & 50 \\
\hline $\begin{array}{l}\text { Total } / 16 \\
\text { Course } 5\end{array}$ & 9 & 3 & 5 & 3 & 6 & 3 & 3 & 2 & 2 & 3 & 13 \\
\hline$\%$ FB Read & 56 & 19 & 31 & 19 & 38 & 19 & 19 & 13 & 13 & 19 & 81 \\
\hline Total/11 & 5 & 4 & 4 & 6 & 4 & 2 & 6 & & & & 6 \\
\hline$\%$ FB Read & 45 & 36 & 36 & 55 & 36 & 18 & 55 & & & & 55 \\
\hline
\end{tabular}

Table 2. Percentage of Feedback Read across Course Assignments- Level 2 Prompt

\begin{tabular}{|c|c|c|c|c|c|c|c|c|c|c|c|}
\hline \# of Students & Mod.1 & Mod.2 & Mod.3 & Mod.4 & Mod.5 & Mod.6 & Mod.7 & Mod.8 & Mod.9 & Mod.10 & Final Exam/Project \\
\hline $\begin{array}{l}\text { Total/15 } \\
\text { Course } 1 \\
\end{array}$ & 8 & 12 & 8 & 7 & 7 & 7 & 4 & 4 & 5 & 4 & 5 \\
\hline$\%$ FB Read & 53 & 80 & 53 & 47 & 47 & 47 & 27 & 27 & 33 & 27 & 33 \\
\hline $\begin{array}{l}\text { Total/18 } \\
\text { Course } 2\end{array}$ & 10 & 15 & 11 & 9 & 8 & 6 & 5 & 4 & 6 & 5 & 8 \\
\hline$\%$ FB Read & 67 & 83 & 73 & 60 & 53 & 40 & 33 & 27 & 40 & 33 & 53 \\
\hline
\end{tabular}


The results confirmed the hypothesis that prompting students to read their feedback (Level 1 group) will improve their online written feedbackreading rate while teaching online courses. This improved result were established by comparing the current results to an original study of Sawicki and O'Rourke [9] which showed a 34\% feedback- reading rate with little or no prompts. The Level 1 group in the current research improved the feedback reading to $39 \%$ with Announcement post prompts. To further improve the students' reading their online feedback the instructor required the students to read and reflect on their feedback for a numerical grade (Level 2 Group). The Level 2 prompt improved the reading rate to $82 \%$ on an individual assignment were a grade was given for reading and reflecting on their feedback. Requiring students to read and reflect on their assignment written reading improved the overall course feedback-reading rate to $44 \%$. This represents a $10 \%$ increase over the original study by Sawicki and O’Rourke [9] when no prompts were provided.

Other interesting findings were shown in the results. The students in the Level 1 and Level 2 groups both reviewed early course feedback more than later feedback in the course. Viewing the Tables 1 and 2 it is visually evident from the tallies that the students review feedback in the early modules at a higher rate than in the later modules. For example, Level 1 Course 3 Module 1 (Table 1) students reviewed their feedback at a $78 \%$ frequency and Level 2 Course 1 Module 3 (Table 2) students reviewed their feedback at a $53 \%$ reading feedback frequency rate in the first course and $73 \%$ in the second course. The first five modules in Table 2 for the two groups of students showed a $58 \%$ reading feedback frequency rate which an improvement to $44 \%$ overall reading feedback rate. Visually reviewing Tables 1 and 2 it is evident students read their online written feedback more often early in the course than they do later in the course. This supports the findings of the original study by Sawicki and O'Rourke [9].

Another interesting finding was that students in the Level 1 feedback prompt group read their major assignment feedback at a rate of $66 \%$ and the Level 2 feedback prompt group at a rate of $44 \%$. Both represent a marked improvement from the original study of Sawicki and O'Rourke [9] who showed students read their major assignment feedbacks (without prompts) at a rate of $35 \%$. This research indicates that instructors should use Announcement posts and remind students that online written feedback is available to them at all times. A prompt of any sort will improve students reading feedback rate as was shown in the major assignment feedback- reading rate as shown in both the Level 1 and Level 2 prompt groups.

A further finding was that before and after the Module which required students to read and reflect on their online feedback for a grade (Level 2 group), the feedback reading rate improved to $53 \%$ in one group and $73 \%$ in the other group. This indicates that requiring students to read and reflect on their online written feedback for a grade will improve their reading rates after the required module. Consideration by the instructor may be to require students to read their online written for a numerical grade on several assignments during this semester. It is speculated this would be improve feedback reading rates after the module used for grading plus increase the overall student feedback reading rate.

A final anecdotal finding came as a result of one of the researchers withholding the grades from the student grade book so students would have to go into their drop box (the place on the web site where the assignments are submitted) and read their online written feedback along with seeing their numerical grade. By performing a hand count of the tallies of feedbacks read throughout the course, it was shown that $80 \%$ of students read their online written feedback with the numerical grade attached to it. This is quite an astounding result when you consider that if the students do not go into their drop box and read their written feedback and receive a numerical grade, they will have no idea how they are doing in the course. It was shown that $20 \%$ of the time that the students actually took the course without knowing how they did on assignments, either in written form or numerical form. Further, research will be done to investigate why this finding occurred.

\section{Conclusion}

The research looked at improving the rate that students read their online feedback from an earlier study by Sawicki and O'Rourke [9] which showed $34 \%$ of students read their online written feedback. The current study found that requiring students to read and reflect on their online written feedback for a grade can result in students reading their feedback $80 \%$ of the time. The overall course feedback reading rate for Level 2 feedback prompts was $44 \%$ which was an improvement over the $39 \%$ reading rate in the Level 1 group (Announcement post prompt) and the 34\% without prompts (original study Sawicki and O'Rourke [9]). Instructors will have to consider if or how often they will require students to read their online written feedback for a grade. It is shown in this study the more an instructor requires students to read their online written feedback the higher compliance amongst students will occur. The $80 \%$ rate of reading online written feedback when it is required for a grade is a high reading rate but is somewhat perplexing and it can be speculated that some students do not know how to look at their online written feedback or know where to look for it or they do not see it as useful. This supports the findings of Careless [1].

In van der Pol, van der Berg, Admiraal and Simons [11] it was shown that there was a high correlation 
between how useful students felt the feedback was the more the receiver agreed with the feedback. The concept of how useful the students find the online written feedback may be important in the current study and might have implications for the current results. Future studies directed at finding out how useful the students perceive the online written feedback should be investigated along with other ways to improve students reading their online written.

\section{References}

[1] Careless, D., (2006). Differing perceptions in the feedback process. Studies in Higher Education, 31 (2), 219233.

[2] Cavanaugh, L. and Sawicki, T.M., (2013). Perceptions of Taking an Online Physical Education Courses: A Qualitative Analysis. International Journal of Instructional Technology and Distance Learning, 10 (5), 65-71.

[3] Coll, C., Rochera, M.J, and de Gispert, I., (2014). Supporting online collaborative learning in small groups: teacher feedback on learning content, academic task and social participation. Computers and Education, 75, 53-64.

[4] Dysthe, O., Lillejord, S., Vines, A. and Wasson, B., (2010). Productive e-feedback in higher education. Some critical issues. In Lugvigsen, S., Lund, A., Rasmussen, I., and Saljo, R. (Ed.). Learning across sites: New tools, infrastructures and practices. Pergamon Press, Oxford, UK.

[5] Gikandi, J.W., Morrow, D. and Davis, N.E., (2011). Online formative assessment in higher education: a review of literature. Computers and Education, 57, 2333-2351.

[6] Mandernach, J., and Garrett, J., (2016). Effective feedback strategies for the online classroom, Retrieved from https://www.facultyfocus.com/resources/online-learning/ grading-and-feedback-online-learning/effective-feedba ckstrategies-online-classroom-2/ (Access Date: 4 June, 2017).
[7] Murphy, C., (2010). Student perceptions of feedback: seeking a coherent flow. Practitioner Research in Higher Education, 4 (1), 41-51.

[8] Ramsden, P., (2003). Learning to teach in higher education (2nd edition). London, Routledge Falmer.

[9] Sawicki, T. M. and O'Rourke, S., (2016). Use of Student Feedback for Online Learning. The International Journal for Digital Society (IJDS), Vol. 7, (2): 1152 - 1156.

[10] Strong, J., Hughes, C., Wilson., Arnott, W., Isles, R. and Bennison, A., (2012). Perceptions of Feedback among Undergraduate and Postgraduate Students of Four Health Science Disciplines. The Internet Journal of Allied Health Sciences and Practice, 10(4), 1-8.

[11] van der Pol, J, van der Berg, B.A.M., Admiraal, W.F. and Simons, P.R.J., (2008). The nature, reception, and use of online peer feedback in higher education. Computers and Education, 51 (4), 1804-1817.

[12] Zhu, E., (2006). Interaction and cognitive engagement: an analysis of four asynchronous online discussions. Instructional Science, 34, 451-480. 